\title{
Effects of 7-week shoulder exercise intervention on joint mobility, strength and function in diabetics and non-diabetics
}

\begin{abstract}
Objective of the study was to compare the effects of a 7-week shoulder exercise program on shoulder range of motion(ROM), strength, and function between diabetics/pre-diabetics and non-diabetics. Shoulder ROM (internal rotation(IR) and external rotation(ER)), strength (elevation, retraction, IR, and ER), and function were assessed pre/post intervention. Shoulder function was assessed using Disabilities of the Arm, Shoulder, and Hand(DASH) and Shoulder Pain and Disability Index(SPADI). Improvements in variables were compared between groups using mix-model ANOVAs. Two pre-diabetics participants developed shoulder pain and were excluded from analysis. These participants were significantly older compared to the rest of the participants (69.0 vs. 55.7 years). After excluding these participants, there were no significant group differences (7diabetics/pre-diabetics vs. 8 non-diabetics) in intervention effects. In both groups, intervention resulted in improved ER $\operatorname{ROM}(\mathrm{p}=.002), \operatorname{IR}(\mathrm{p}=.004)$ and ER strength $(\mathrm{p}=.010), \operatorname{DASH}(\mathrm{p}=.010)$ and $\operatorname{SPADI}(\mathrm{p}=.025)$ scores. Shoulder exercises improved shoulder mobility, strength, and function in diabetics and non-diabetics alike. Introduction of shoulder exercises appears to be beneficial in improving shoulder function, regardless of diabetes status. However, caution may be needed when working with older diabetic patients, as they may be more prone to developing shoulder pain.
\end{abstract}

Keywords: shoulder pain, diabetes, rehabilitation and intervention.
Volume 3 Issue I - 2018

Sakiko Oyama,'Araceli Sosa,' Jose Romero²

'Department of Kinesiology, Health and Nutrition, University of Texas at San Antonio, USA

${ }^{2}$ Health Educator, University Health Service, USA

Correspondence: Sakiko Oyama, Department of Kinesiology, Health, and Nutrition, University of Texas at San Antonio, USA, Tel 210-458-5435, Fax 210-458-5873,

Email sakiko.oyama@utsa.edu

Received: December 19, 2017 | Published: January 22, 2018
Abbreviations: ROM, range of motion; IR, internal rotation; ER, external rotation; DASH, disabilities of the arm, shoulder and hand; SPADI, shoulder pain and disability index

\section{Introduction}

In the United States, the prevalence of diabetes has increased from $5.5 \%$ to $9.3 \%$ within past decades ${ }^{1}$ with the disease currently affecting 29.1 million people. ${ }^{2}$ Diabetes is commonly associated with conditions such as cardiovascular disease and long-term negative effects on various organs in the body such as kidneys and eyes. ${ }^{3}$ However, diabetes has also been shown to affect the musculoskeletal system. ${ }^{4-9}$ Diabetics are four times more likely to have musculoskeletal disorders compared to non-diabetics ${ }^{6}$ with the shoulder being one of the joints most affected by the disease. ${ }^{10}$ Previous studies show that diabetics have higher prevalence of shoulder pain and injuries compared to non-diabetics. ${ }^{6,7,10-14}$ Specifically, diabetics are 3-9 times more likely to have frozen capsulitis and 5 times more likely to have rotator cuff tears compared to non-diabetics. ${ }^{4,12,15}$ Additionally, diabetics are reported to have thickened supraspinatus and biceps tendons ${ }^{16}$ along with decreased ROM in shoulder abduction and flexion ${ }^{4,5}$ an impaired tendon-bone healing capacity ${ }^{17}$ and difficulty regaining range of motion (ROM) after a surgery. ${ }^{18,19}$

The musculoskeletal symptoms described above are attributed to mechanical changes in connective tissue due to formation of advanced glycation end products (AGEs). ${ }^{4}$ The AGEs are formed from a reaction that occurs from bonding of sugar and amino acid. ${ }^{4}$ The AGEs cause increased crosslinking between collagen fibers and increase stiffness and brittleness of the connective tissue. This change in mechanical property makes the tissue more susceptible to failure under stress. ${ }^{20}$ While accumulation of AGEs naturally occurs with aging, the process is accelerated in diabetics due to high blood glucose concentration and decreased peripheral blood flow. ${ }^{4}$ The insertion of the rotator cuff on the greater tubercle corresponds to an area of poor blood supply ${ }^{21-23}$ which may further contribute to accumulation of AGEs.

Stretching and strengthening exercises focused on improving rotator cuff strength and restoring balance between the muscles that stabilize and rotate scapula are used in prevention and treatment of shoulder pain. ${ }^{21-28}$ Effectiveness of this approach has been demonstrated in both healthy individuals and patients with various shoulder injuries. Despite the increased prevalence of shoulder pain among diabetics, there has only been one study that examined the effect of a physiotherapy program specifically in this population. ${ }^{29}$ The study demonstrated that implementation of a physiotherapy program resulted in decreased pain level and improved shoulder function in diabetic patients who had shoulder pain. ${ }^{29}$ However, this study did not have a comparison group without diabetes; therefore it is unknown how changes in tissue property caused by diabetes moderates the effectiveness of strengthening and stretching exercises. Furthermore, the participants in the study had moderate to severe shoulder pain at baseline, thus improvements in pain level and function they observed may be due to natural recovery. It would be beneficial for clinicians working with diabetic patients to know how performing exercises can affect their shoulder mobility, strength, and function, and whether these effects are affected by diabetes. Such data would help clinicians set realistic rehabilitation goals and evaluate effectiveness of treatment. Therefore, the purpose of this study was to compare the effects of a 7-week shoulder exercise program on shoulder ROM, strength, and 
perceived shoulder function and disability between diabetics/prediabetics and non-diabetics.

\section{Materials and methods}

\section{Participants}

A total of 11 diabetics or pre-diabetics and 10 non-diabetics participated in this study. Diagnosis of diabetes/pre-diabetes was made by physicians based on 2014 American Diabetes Association's Standards of Care. ${ }^{3}$ Diabetics and pre-diabetics were grouped together in this study, due to a small sample size. The demographics of the participants are described in Table 1. All participants were enrolled in a health education/exercise program offered through the local hospital system (Texas Diabetes Institute). The program is offered to individuals with variety of chronic diseases, including diabetes. Participants who had shoulder pain that kept them from providing full effort during the baseline strength testing and those who developed shoulder pain while in the study were excluded from the analysis. All participants with minimum attendance rate of $70 \%$ were included in the analysis. The study protocol was approved by the University of Texas Health Science Center at San Antonio Institutional Review Board and the University Health System.

Table I The demographics of the participants are described

\begin{tabular}{lll}
\hline & Non-diabetic $(\mathbf{n}=8)$ & $\begin{array}{l}\text { Diabetic/pre- } \\
\text { diabetics(n=7) }\end{array}$ \\
\hline Age(years) & $56.6 \pm 10.4$ & $56.0 \pm 5.7$ \\
Body Mass(kg) & $158.4 \pm 11.1$ & $167.4 \pm 36.8$ \\
Height $(\mathrm{cm})$ & $92.9 \pm 18.6$ & $119.0 \pm 15.0$ \\
BMI & $37.8 \pm 10.4$ & $42.5 \pm 4.00$ \\
\hline
\end{tabular}

\section{Procedure}

The health education program consisted of 45 minutes of health education class followed by 45 minutes of physical activity held twice a week for ten weeks. A total of seven strengthening exercises using resistive bands, and three stretching exercises were incorporated into the physical activity portion of the program (Figure 1). During the first two weeks of the program, informed consent was obtained, baseline data collection was completed and participants were instructed on proper exercise technique. The participants performed the exercises twice a week for the next seven weeks as a part of the program. The post-intervention data collection was conducted during the last week of the program.

\section{Data collection}

Shoulder internal and external rotation range of motion: Shoulder internal rotation and external rotation ROM was assessed using a digital inclinometer (Baseline, Fabrication Enterprises, Inc., White planes, NY).$^{30}$ Initially, we intended to include measurement of shoulder flexion ROM. However, the measurement was eliminated since the supine measurement of shoulder flexion ROM required participants to lay at the edge of the portable treatment table which involved risk of table tipping over or patient falling off the table. The inclinometer measures angle in degrees in a vertical plane. ${ }^{30}$ The internal and external rotation ROM was assessed while participants lay supine on a treatment table and shoulder abducted and elbow flexed to $90^{\circ}$. One examiner passively moved participant's shoulder into end internal and external rotation $\mathrm{ROM}$ while applying downward force on the anterior aspect of the shoulder to stabilize the scapula. The second examiner measured ROM using a digital inclinometer. The measurements were taken three times on participant's dominant shoulder. The intersession (between day) intra-class correlation coefficient (ICC) for internal rotation and external rotation established prior to the study were .924 and .876 , respectively. The standard error of measurement for internal rotation and external rotation were $3.1^{\circ}$, and $3.2^{\circ}$, respectively.

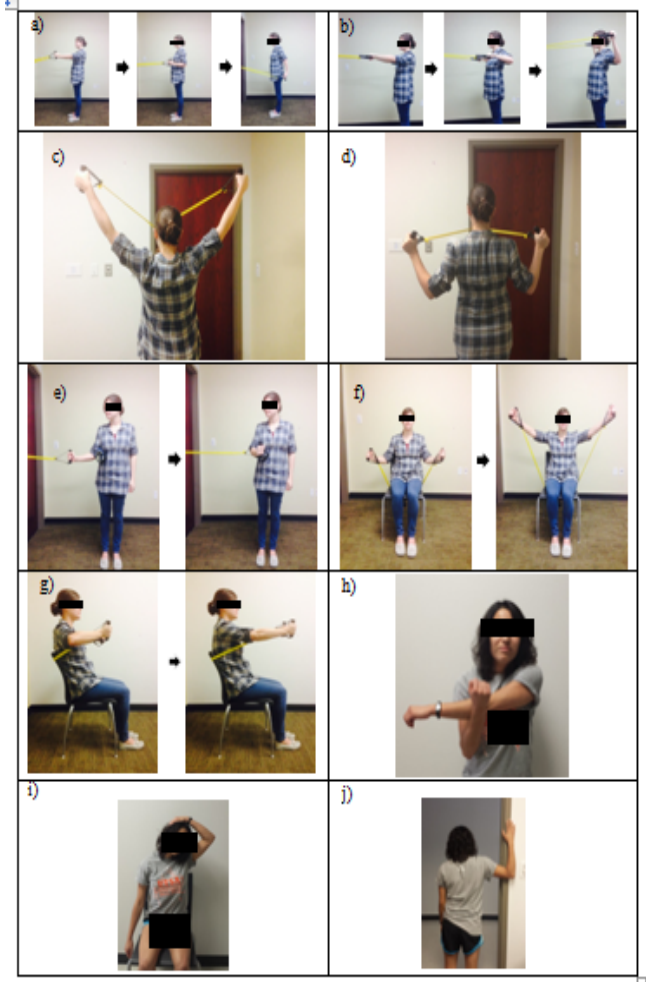

Figure I Exercises performed in this study. A) Low row with arm extension, participants pulled elbows to their side, and then extended their elbow. B) " $T$ " with rotation, keeping elbows at shoulder level, participants pulled elbows backwards (horizontal abduction) while retracting the scapula, and then externally rotated their shoulders. C) "Y", keeping their elbows straight, participants pulled their arms backward in a shape of a letter " $Y$ " while retracting the scapula. D) "W", keeping their shoulders elevated $45^{\circ}$ and elbows flexed $90^{\circ}$, participants pulled their arms backward in a shape of a letter "W" while retracting the scapula. E) Internal rotation, keeping their elbows flexed $90^{\circ}$, participants rotated their shoulder into internal rotation, F) Elevation, sitting in a chair, participants elevated their arms as high as they could. G) Punch with plus, sitting in a chair, participants extended their elbow as if punching forward, and then protracted their scapular at the end of the motion. H) Cross-chest stretch, participants brought one arm across their chest and used the other arm to push it into horizontal adduction to stretch posterior shoulder structures. I) Neck stretch, using one arm, participants laterally flexed their neck towards the side of the arm to stretch neck and upper trapezius muscles. J) Door way pec stretch, using a door way or a wall, participants horizontally abducted their arm to stretch pectoral muscles.

Shoulder strength: Shoulder elevation, retraction, shoulder internal rotation, and external rotation strength were measured using a handheld dynamometer (Lafayette Inc., Lafayette, IN). ${ }^{24}$ The muscle strength was measured using a break test. ${ }^{24,31}$ The participants were asked to position the testing limb in a specific position and hold that position against the examiner's resistance. The maximal force was recorded as the examiner "broke" the participant's arm from the testing position. For elevation strength, participants sat in a chair and 
lifted their shoulder to $90^{\circ}$ in a scapular plane $\left(30^{\circ}\right.$ anterior to the frontal plane). The examiner applied a downward force at the wrist. For retraction, the participant sat in a chair and abducted their shoulder to $120^{\circ}$ while retracting their shoulder blades. The examiner applied force at the wrist in anterolateral direction. For internal and external rotation, participants lay supine on the treatment table with their shoulder abducted and elbow flexed to $90^{\circ}$. The examiner stabilized the participant's arm while applying force caudally towards the feet (external rotation) and cranially towards the head (internal rotation). The measurements were taken three times on participant's dominant shoulder. The intersession ICC for elevation, retraction, internal rotation, and external rotation established before the study ranged from .755 to .912 . The standard error of measurement for elevation, retraction, internal rotation, and external rotation ranged from $9.3 \mathrm{~N}$ to $16.2 \mathrm{~N}$.

Shoulder function and disability: Shoulder function and disability was assessed using two self-administered instruments, Disabilities of Arm, Shoulder, and Hand (DASH) and Shoulder Pain and Disability Index (SPADI). DASH is a 30-item questionnaire that is designed to measure physical function and symptoms in people with musculoskeletal disorders of the upper limb. ${ }^{32}$ SPADI includes 13 questions regarding pain severity and ability to perform activities of daily living (ADL). ${ }^{33}$ Both of these instruments are commonly used to assess shoulder pain/function in orthopedic research. ${ }^{14,34-36}$ The data collection procedure was completed at baseline and at the completion of the intervention program by the same examiner. The examiners were blinded to the participants' diabetes status.

Exercise intervention: The exercises program included stretching of the chest, neck, and posterior shoulder muscles, and strengthening exercises that target rotator cuff and muscles around the scapula (Figure 1). ${ }^{24,30,37}$ All exercises were performed using resistive bands (Theraband $^{\circledR}$, Hygenic Corporation, Akron, $\mathrm{OH}$ ) so that the exercises can be performed in any setting. Four levels of resistance bands were used to perform the exercises. The yellow, red, green, and blue resistive bands provided the lowest to highest amount of resistance. At the beginning of the program, each participant selected the appropriate level (color) of resistive bands to be used for each exercise. The appropriate level of resistive band was determined as the band with the highest resistance that participants could use to complete 10 repetitions of the exercise. We used a card that indicated the band color to be used for each exercise. The participants wore the card around his or her neck while performing the exercises, in order to keep track of the appropriate band color for each exercise. During the first 3 weeks (wk1-3), the participants performed 3 sets of 10 repetitions of each exercise, while focusing on the technique and gradually increasing resistance. The repetitions were increased to $12-15$ over the next 2 weeks (wk45) and to 15-20 over the last 2 weeks of the program (wk6-7). Each stretching exercise was held for 15 seconds, and was repeated three times on each side. In addition to performing the exercises in the program twice a week, the participants were encouraged to perform the exercises at least once during a week on their own using the resistive bands provided. Attendance was taken during each session, and days on which the participants performed exercises on their own at home were recorded on the journal that they kept as a part of the program.

Data analysis: Percent changes in shoulder ROM, strength, and function after the intervention in diabetics and non-diabetics were compared using an independent t-test. Additionally, shoulder ROM, strength, and function before and after the intervention for the whole group were compared using Wilcoxon signed rank test. SPSS Statistical analysis software (Version 22) was used for data analysis. A priori alpha level was set to 0.05 .

\section{Results and discussion}

\section{Results}

Three participants (1 pre-diabetic and 2 non-diabetic) dropped out of the study for non-study related reasons immediately after the baseline, and one diabetic participant was excluded from the study due to low compliance. Two pre-diabetic participants were excluded from the study due to development of shoulder pain that disabled them from continuing to perform the exercises and perform postintervention strength testing. These participants were older (69.0 099.9 years) compared to the rest of the participants (55.7 7.9 years).

After excluding the above participants, we were left with 7 participants in the diabetes/pre-diabetes group ( 2 males $/ 5$ females, age: $56.0 \pm 5.7$ years, height: $167.4 \pm 6.8 \mathrm{~cm}$, Mass: $119.0 \pm 15.0 \mathrm{~kg}$, DASH: $22.5 \pm 22.0$, SPADI: $29.0 \pm 29.8$ ) and 8 participants in the non-diabetic group ( $1 \mathrm{male} / 7$ females, age: $56.6 \pm 10.4$ years, height: $158.4 \pm 11.1 \mathrm{~cm}$, Mass: $92.9 \pm 18.6 \mathrm{~kg}$, DASH: $23.5 \pm 19.6$, SPADI: $23.3 \pm 20.3$ ). Diabetes/ pre-diabetes group participants had significantly greater body mass compared to the non-diabetic participants (Mean difference: $26.1 \mathrm{~kg}$, $\mathrm{p}=.011)$. Other demographics and pre-intervention DASH and SPADI scores were not statistically different between groups. The normal distribution and equal variance of the percent change variables were confirmed using the Shapiro-Wilk test and Levene's test. There were no significant group differences ( 7 diabetics /pre-diabetics vs. 8 nondiabetics) in intervention effects. Since there were no difference in the intervention effects between groups, data from the two groups were pooled to examine the effects of intervention across the groups using the Wilcoxon Signed Rank Test. Non-parametric statistics was used here since many of the variables were not normally distributed due to the small sample size. Intervention resulted in improved ER ROM $(\mathrm{p}=.003), \mathrm{IR}(\mathrm{p}=.005)$ and ER strength $(\mathrm{p}=.023)$, DASH $(\mathrm{p}=.013)$, and SPADI $(p=.034)$ scores across the groups (Table 2$)$.

\section{Discussion}

The purpose of this study was to compare the effects of a 7-week shoulder exercise program on shoulder ROM, strength, and perceived shoulder function and disability between diabetics/pre-diabetics and non-diabetics. We observed that the exercise program resulted in improved shoulder external rotation ROM, shoulder rotation strength, and shoulder function in participants with and without diabetes alike.

Maintaining adequate shoulder rotation ROM is important in performing ADLs. For example, in order to touch back of head or neck, as if washing hair, at least 50 degrees of shoulder external rotation is required. ${ }^{38}$ Our shoulder exercise program included stretching exercises that are aimed to improve shoulder internal rotation ROM (cross-arm stretch) and external rotation ROM (doorway stretch). ${ }^{30,39,40}$ However, we saw improvement in ER ROM, but not in IR. A previous study on healthy females reported that the shoulder ER ROM decreased with aging, but that IR ROM was unaffected by aging. ${ }^{41}$ Perhaps, our participant's ER ROM was decreased at baseline due to aging, and thus had room for improvement, whereas IR ROM was not limited to begin with, and thus did not have much room for improvement. 
Weakness of the shoulder rotator muscles is associated with shoulder pain. ${ }^{42,43}$ In particular, infraspinatus and teres minor that act to externally rotate the shoulder and subscapularis that acts to internally rotate the shoulder are part of the rotator cuff that functions to stabilize the glenohumeral joint through concavity compression. ${ }^{44,45}$ Weakness in these muscles would lead to compromised glenohumeral differences (7 diabetics / pre-diabetics vs. 8 non-diabetics) in intervention effects. In both groups, intervention resulted in improved ER ROM ( $p=.002), I R(p=.004)$ and ER strength $(\mathrm{p}=.010)$, DASH $(\mathrm{p}=.010)$, and SPADI $(\mathrm{p}=.025)$ scores

\begin{tabular}{lllll}
\hline & \multicolumn{2}{l}{ Non diabetics $(\mathbf{N}=8)$} & \multicolumn{2}{l}{ Diabetics/pre-diabetics $(\mathbf{N}=7)$} \\
\cline { 2 - 5 } & Pre & Post & Pre & Post \\
\hline Range of motion $\left(^{\circ}\right)$ & & & \\
Internal rotation & $47.6 \pm 7.8$ & $53.0 \pm 10.5$ & $42.0 \pm 8.5$ & $45.6 \pm 3.8$ \\
External rotation & $97.3 \pm 18.3$ & $109.6 \pm 15.0$ & $113.7 \pm 17.2$ & $124.5 \pm 10.8$ \\
Strength (\%BW) & & & & \\
Internal rotation & $111.0 \pm 37.2$ & $124.2 \pm 29.4$ & $120.6 \pm 51.7$ & $141.6 \pm 62.2$ \\
External rotation & $114.3 \pm 33.2$ & $125.1 \pm 26.9$ & $115.1 \pm 32.0$ & $133.2 \pm 48.0$ \\
Elevation & $67.6 \pm 30.9$ & $74.7 \pm 27.5$ & $76.4 \pm 31.2$ & $82.4 \pm 39.9$ \\
Retraction & $73.6 \pm 28.3$ & $79.3 \pm 24.4$ & $84.4 \pm 22.6$ & $89.9 \pm 36.2$ \\
Functional scores $(\mathbf{p t s})$ & & & \\
DASH & $23.5 \pm 19.6$ & $11.5 \pm 9.7$ & $22.0 \pm 20.1$ & $18.6 \pm 24.2$ \\
SPADI & $23.3 \pm 20.3$ & $15.0 \pm 18.6$ & $29.0 \pm 29.8$ & $17.0 \pm 18.8$ \\
\hline
\end{tabular}

DASH, disability of arm, shoulder, and hand; SPADI, shoulder pain and disability index

Intervention used in our study resulted in improved shoulder rotation strength, but no change in shoulder elevation or scapular retraction strength. Lack of increase in shoulder elevation strength may be explained by the fact that only 1 of the 7 strengthening exercises targeted the shoulder elevator muscles (elevation). On the other hand, lack of improvement in scapular retraction was unexpected, since scapular retraction was emphasized in most of the strengthening exercises in the intervention (Ys, Ts, Ws, elevation, rows). This observation may be attributed to the relatively low reliability of the measurement for the scapular retraction strength, which may be attributed to difficulty in measuring scapular retraction strength without crossing multiple joints. The testing procedure we used is a common way to assess scapular retraction strength. ${ }^{24,31,43,53}$ However, in a study that examined the effects of exercise program on scapular retraction strength in intercollegiate swimmers, participants' retraction strength measured using the same method (i.e. arm in $120^{\circ}$ of elevation) did not improve with intervention, while the retraction strength measured with shoulder at a lower elevation angle $\left(90^{\circ}\right.$ of elevation) improved. ${ }^{24}$ It is possible that selecting a different variation of strength testing may have allowed us to demonstrate the improvement in retraction strength.

The exercise intervention resulted in an increased functional score (DASH) and a decreased disability score (SPADI). These improvements are meaningful in that the participants who were included in the analysis did not have significant shoulder pain to begin with. While many of the participants complained of stiffness and general discomfort with extreme shoulder movements, none of them had shoulder pain that was severe enough to keep them from completing the baseline ROM and strength testing. The baseline score joint stability, and thus increased risk of various shoulder pathologies, including rotator cuff tear. ${ }^{44,46}$ Similarly, weakness of the muscles that control scapular movement has been linked to shoulder pain. ${ }^{47,48}$ Weakness or decreased activity of the muscles can result in scapular dyskinesis ${ }^{49,50}$ which can increase impingement of the supraspinatus and long head of the biceps tendons within the subacromial space. ${ }^{51,52}$ 
participants dropped out from the study, because they developed shoulder pain during the intervention. For the participants who developed pain during the intervention, it is difficult to determine if the shoulder pain resulted from the shoulder exercise intervention or their activity outside of the intervention. If the intervention were to blame, it would suggest that exercise selection and intensity would have to be modified for diabetic/pre-diabetic patients. However, we noticed that the two diabetic/pre-diabetic patients who dropped out of the intervention, and the two that were not included in the study due to shoulder pain were significantly older compared to the rest of the participants. The average age of the 4 participants were $69.8 \pm 7.8$ years, while the average age of the rest of the participants were $55.1 \pm 8.2$ years. Since it has been demonstrated that prevalence of shoulder pain increases with age $\mathrm{e}^{54,57,58}$ shoulder pain in these participants may be simply attributed to their age.

Participation in regular cardiovascular activities, such as walking and jogging, is recommended for diabetics to help with weight loss, management of blood glucose level, and to improve cardiovascular health. While these exercises are important, these activities do not involve significant upper extremity movements. Considering the high prevalence of shoulder pain and stiffness in diabetic patients, it may be recommended for the patients to incorporate regular shoulder exercises into their routine. Maintenance of adequate shoulder mobility and strength are necessary to perform activities of daily living and other activities (i.e. hobbies, sports, and work) that enhance quality of life. Prevalence of shoulder pain increases with age regardless of diabetes ${ }^{54,57,58}$ Therefore, starting a shoulder exercise routine earlier in life may help prevent or delay the development of shoulder pain later on in life.

There are a few limitations that we acknowledge. The small sample size of the study precluded us from running an analysis that takes potential modifiers, such as age, disease state, and prior history of injury into account. Furthermore, the participants in the 2 groups were not matched based on their age, gender, and baseline strength level, which may have potentially confounded the results. This should be addressed in a larger study in the future. Due to the small sample size, we had to group pre-diabetic and diabetic participants into a single group. While this may be a limitation, the values representing strength, mobility, and function were very similar between the prediabetics and diabetics. The participants were supervised during the exercise programs, but not during the home exercise program. However, the home exercise program was not the main focus of this study. It is meaningful that improvements resulted even without the supervised home exercise program.

\section{Conclusion}

Shoulder exercises improved shoulder mobility, strength, and function in diabetics and non-diabetics alike. Introduction of shoulder exercises is beneficial in improving shoulder function, regardless of diabetes status. However, caution may be needed when working with older diabetic patients, as they are more prone to developing shoulder pain.

\section{Acknowledgements}

This study was funded by the University of Texas at San Antonio College of Education Faculty Research Award.

\section{Conflict of interest}

There is no conflict of interest to disclose.

\section{References}

1. Selvin E, Parrinello CM, Sacks DB, et al. Trends in prevalence and control of diabetes in the United States, 1988-1994 and 1999-2010. Annals of internal medicine. 2014;160(8):517-525.

2. National diabetes statistics report. USA: Diabetes public health resource; 2014.

3. Association AD. Standards of medical care in diabetes-2014. Diabetes Care. 2014;37(Suppl 1):S14-80.

4. Abate M, Schiavone C, Pelotti P, et al. Limited joint mobility (LJM) in elderly subjects with type II diabetes mellitus. Arch Gerontol Geriatr. 2011;53(2):135-140.

5. Balci N, Balci MK, Tuzuner S. Shoulder adhesive capsulitis and shoulder range of motion in type II diabetes mellitus: association with diabetic complications. J Diabetes Complications. 1999;13(3):135-140.

6. Cagliero E, Apruzzese W, Perlmutter GS, et al. Musculoskeletal disorders of the hand and shoulder in patients with diabetes mellitus. Am J Med. 2002;112(6):487-490.

7. Cole A, Gill TK, Shanahan EM, et al. Is diabetes associated with shoulder pain or stiffness? Results from a population based study. J Rheumatol. 2009;36(2):371-377.

8. Huang YP, Fann CY, Chiu YH, et al. Association of diabetes mellitus with the risk of developing adhesive capsulitis of the shoulder: a longitudinal population-based followup study. Arthritis care \& research. 2013;65(7):1197-1202.

9. Shah KM, Clark BR, McGill JB, et al, Upper extremity impairments, pain and disability in patients with diabetes mellitus. Physiotherapy. 2015;101(2):147-154.

10. Molsted S, Tribler J, Snorgaard O. Musculoskeletal pain in patients with type 2 diabetes. Diabetes research and clinical practice. 2012;96(2):135140 .

11. Hayes MJ, Smith DR, Taylor JA. Musculoskeletal disorders and symptom severity among Australian dental hygienists. BMC research notes. $2013 ; 6: 250$.

12. Thomas SJ, McDougall C, Brown ID, et al. Prevalence of symptoms and signs of shoulder problems in people with diabetes mellitus. J Shoulder Elbow Surg. 2007;16(6):748-751.

13. Siu DC, Tse LA, Yu IT, et al. Computer products usage and prevalence of computer related musculoskeletal discomfort among adolescents. Work. 2009;34(4):449-454.

14. Wilk KE, Macrina LC, Fleisig GS, et al. Deficits in glenohumeral passive range of motion increase risk of elbow injury in professional baseball pitchers: a prospective study. Am J Sports Med. 2014;42(9):2075-2081.

15. Kang JH, Tseng SH, Jaw FS, et al. Comparison of ultrasonographic findings of the rotator cuff between diabetic and nondiabetic patients with chronic shoulder pain: a retrospective study. Ultrasound Med Biol. 2010;36(11):1792-1796.

16. Abate M, Schiavone C, Salini V. Sonographic evaluation of the shoulder in asymptomatic elderly subjects with diabetes. BMC musculoskeletal disorders. 2010;11:278.

17. Bedi A, Fox AJ, Harris PE, et al. Diabetes mellitus impairs tendon-bone healing after rotator cuff repair. J Shoulder Elbow Surg. 2010;19(7):978988.

18. Abate M, Schiavone C, Salini V, et al. Management of limited joint mobility in diabetic patients. Diabetes, metabolic syndrome and obesity targets and therapy. 2013;6:197-207.

19. Cinar M, Akpinar S, Derincek A, et al. Comparison of arthroscopic capsular release in diabetic and idiopathic frozen shoulder patients. Arch Orthop Trauma Surg. 2010;130(3):401-406. 
20. DeGroot J. The AGE of the matrix: chemistry, consequence and cure. Current opinion in pharmacology. 2004;4(3):301-305.

21. Ling SC, Chen CF, Wan RX. A study on the vascular supply of the supraspinatus tendon. Surg Radiol Anat. 1990;12(3):161-165.

22. Lohr JF, Uhthoff HK. The microvascular pattern of the supraspinatus tendon. Clin Orthop Relat Res. 1990;(254):35-38.

23. Rathbun JB, Macnab I. The microvascular pattern of the rotator cuff. $J$ Bone Joint Surg Br. 1970;52(3):540-553.

24. Hibberd EE, Oyama S, Spang JT, et al. Effect of a 6-week strengthening program on shoulder and scapular-stabilizer strength and scapular kinematics in division i collegiate swimmers. J Sport Rehabil. 2012;21(3):253-265.

25. Andersen LL, Christensen KB, Holtermann A, et al. Effect of physical exercise interventions on musculoskeletal pain in all body regions among office workers: a one-year randomized controlled trial. Man Ther. 2010;15(1):100-104.

26. Andersen LL, Jorgensen MB, Blangsted AK, et al. A randomized controlled intervention trial to relieve and prevent neck/shoulder pain. $\mathrm{Med} \mathrm{Sci}$ Sports Exerc. 2008;40(6):983-990.

27. Andersen LL, Saervoll CA, Mortensen OS, et al. Effectiveness of small daily amounts of progressive resistance training for frequent neck/shoulder pain: randomised controlled trial. Pain. 2011;152(2):440-446.

28. Lynch SS, Thigpen CA, Mihalik JP, et al. The effects of an exercise intervention on forward head and rounded shoulder postures in elite swimmers. Br J Sports Med. 2010;44(5):376-381.

29. Kyhlback M, Schroder Winter H, Thierfelder T, et al. Physiotherapy treatment of the diabetic shoulder: a longitudinal study following patients with diabetes and shoulder pain using a pre-post treatment design. Disabil Rehabil. 2014;36(7):556-562.

30. Oyama S, Goerger CP, Goerger BM, et al. Non-assisted posterior shoulder stretches acutely improve shoulder range of motion in collegiate baseball pitchers. Athletic Training \& Sports Health Care. 2010;2(14):163-170.

31. Kendall FP, McCreary EK. Muscles: Testing and Function. 3rd ed. USA: Williams and Wilkins; 1983

32. Elliott BC, Alderson JA, Denver ER. System and modelling errors in motion analysis: implications for the measurement of the elbow angle in cricket bowling. J Biomech. 2007;40(12):2679-2685.

33. Xu X, Chang CC, Faber GS, et al. The validity and interrater reliability of video-based posture observation during asymmetric lifting tasks. Hum Factors. 2011;53(4):371-382.

34. Dixon D, Johnston M, McQueen M, et al. The disabilities of the arm, shoulder and hand questionnaire (DASH) can measure the impairment, activity limitations and participation restriction constructs from the international classification of functioning, disability and health (ICF). BMC musculoskeletal disorders. 2008;9:114.

35. Jester A, Harth A, Wind G, et al. Disabilities of the arm, shoulder and hand (DASH) questionnaire: Determining functional activity profiles in patients with upper extremity disorders. J Hand Surg Br. 2005;30(1):2328.

36. Dodd DJ, Alvar BA. Analysis of acute explosive training modalities to improve lower-body power in baseball players. J Strength Cond Res. 2007;21(4):1177-1182.

37. Oyama S, Myers JB, Wassinger CA, et al. Three-dimensional scapular and clavicular kinematics and scapular muscle activity during retraction exercises. J Orthop Sports Phys Ther. 2010;40(3):169-179.

38. Sheikhzadeh A, Yoon J, Pinto VJ, et al. Three-dimensional motion of the scapula and shoulder during activities of daily living. J Shoulder Elbow Surg. 2008;17(6):936-942.
39. Borstad JD, Ludewig PM. Comparison of three stretches for the pectoralis minor muscle. J Shoulder Elbow Surg. 2006;15(3):324-330.

40. Laudner KG, Sipes RC, Wilson JT. The acute effects of sleeper stretches on shoulder range of motion. J Athl Train. 2008;43(4):359-363.

41. Li X, Ma R, Zhou H, Hanbing Z, et al. Evaluation of hip internal and external rotation range of Motion as an injury risk factor for hip, Abdominal and groin injuries in professional baseball players. Orthopedic reviews. $2015 ; 7(4): 6142$.

42. McClure PW, Michener LA, Karduna AR. Shoulder function and 3-dimensional scapular kinematics in people with and without shoulder impingement syndrome. Phys Ther. 2006;86(8):1075-1090.

43. Trakis JE, McHugh MP, Caracciolo PA, et al. Muscle strength and range of motion in adolescent pitchers with throwing-related pain: implications for injury prevention. Am J Sports Med. 2008;36(11):2173-2178.

44. Lee SB, Kim KJ, O’Driscoll SW, et al. Dynamic glenohumeral stability provided by the rotator cuff muscles in the mid-range and end-range of motion. A study in cadavera. J Bone Joint Surg Am. 2000;82(6):849-857.

45. Labriola JE, Lee TQ, Debski RE, et al. Stability and instability of the glenohumeral joint: the role of shoulder muscles. J Shoulder Elbow Surg. 2005;14(1 Suppl S):S32-38.

46. Chopp JN, O’Neill JM, Hurley K, et al. Superior humeral head migration occurs after a protocol designed to fatigue the rotator cuff: A radiographic analysis. J Shoulder Elbow Surg. 2010;19(8):1137-1144.

47. Michener LA, Boardman ND, Pidcoe PE, et al. Scapular muscle tests in subjects with shoulder pain and functional loss: reliability and construct validity. Phys Ther. 2005;85(11):1128-1138.

48. Cools AM, Witvrouw EE, Mahieu NN, et al. Isokinetic scapular muscle performance in overhead athletes with and without impingement symptoms. J Athl Train. 2005;40(2):104-110.

49. Ludewig PM, Cook TM. Alterations in shoulder kinematics and associated muscle activity in people with symptoms of shoulder impingement. Phys Ther. 2000;80(3):276-291.

50. Morais FCD, Teixeira SLF, Paula GFR. Scapular muscular activity with shoulder impingement syndrome during lowering of the arms. Clin $J$ Sport Med. 2008;18(2):130-136.

51. Cools AM, Witvrouw EE, Declercq GA, et al. Scapular muscle recruitment patterns: trapezius muscle latency with and without impingement symptoms. Am J Sports Med. 2003;31(4):542-549.

52. McClure PW, Bialker J, Neff N, et al. Shoulder function and 3-dimensional kinematics in people with shoulder impingement syndrome before and after a 6-week exercise program. Phys Ther. 2004;84(9):832-848.

53. Donatelli R, Ellenbecker TS, Ekedahl SR, et al. Assessment of shoulder strength in professional baseball pitchers. J Orthop Sports Phys Ther. 2000;30(9):544-551.

54. Chaudhari AM, McKenzie CS, Pan X, et al. Lumbopelvic control and days missed because of injury in professional baseball pitchers. Am J Sports Med. 2014;42(11):2734-2740.

55. Seagrave RA, Perez L, McQueeney S, et al. Preventive effects of eccentric training on acute hamstring muscle injury in professional baseball Orthop J Sports Med. 2014;2(6):2325967114535351.

56. Cho NS, Moon SC, Jeon JW, et al. The influence of diabetes mellitus on clinical and structural outcomes after arthroscopic rotator cuff repair. Am J Sports Med. 2015;43(4):991-997.

57. Byram IR, Bushnell BD, Dugger K, et al. Preseason shoulder strength measurements in professional baseball pitchers: identifying players at risk for injury. Am J Sports Med. 2010;38(7):1375-1382.

58. Anz AW, Bushnell BD, Griffin LP, et al. Correlation of torque and elbow injury in professional baseball pitchers. Am J Sports Med. 2010;38(7):1368-1374 